\title{
Electronic response of aligned multishell carbon nanotubes
}

\author{
J. M. Pitarke ${ }^{1,2}$ and F. J. García-Vidal ${ }^{3}$ \\ ${ }^{1}$ Materia Kondentsatuaren Fisika Saila, Zientzi Fakultatea, Euskal Herriko Unibertsitatea, 644 Posta kutxatila, 48080 Bilbo, \\ Basque Country, Spain \\ ${ }^{2}$ Donostia International Physics Center (DIPC) and Centro Mixto CSIC-UPV/EHU, Donostia, Basque Country, Spain \\ ${ }^{3}$ Departamento de Física Teórica de la Materia Condensada, Facultad de Ciencias, Universidad Autónoma de Madrid, \\ 28049 Madrid, Spain
}

(Received 20 December 1999; revised manuscript received 1 September 2000; published 26 January 2001)

We report calculations of the effective electronic response of aligned multishell carbon nanotubes. A local graphitelike dielectric tensor is assigned to every point of the multishell tubules, and the effective transverse dielectric function of the composite is computed by solving Maxwell's equations. Calculations of both real and imaginary parts of the effective dielectric function are presented, for various values of the filling fraction and the ratio of the internal and external radii of hollow tubules. Our full calculations indicate that the experimentally measured macroscopic dielectric function of carbon nanotube materials is the result of a strong electromagnetic coupling between the tubes, which cannot be accounted for with the use of simplified effective medium theories. The presence of surface plasmons is investigated, and both optical absorption cross sections and energy-loss spectra of aligned tubules are calculated.

DOI: 10.1103/PhysRevB.63.073404

PACS number(s): 78.66.Sq, 41.20.Jb, 61.46.+w, 73.20.Mf

The so-called multishell carbon nanotubes, with diameters on the order of $10 \mathrm{~nm}$ and lengths reaching up to several microns, are needlelike tubes consisting of a finite number of concentric cylindrical two-dimensional layers of graphite sheet arranged around a central hollow with a constant separation between the layers. ${ }^{1,2}$ Since their discovery, ${ }^{1}$ these tubules have attracted much attention because of their size and their novel structural and electronic properties. ${ }^{3-7}$ An important step in the investigation of these new materials was achieved when composites were manufactured with nanotubes organized into well-aligned arrays, ${ }^{8,9}$ since alignment of nanotubes was expected to be important not only to test properties but also for potential applications such as atomicscale field emitters. With the availability of aligned carbon nanotube films, optical measurements were carried out with polarized light, 9,10 thereby evaluating the frequencydependent effective dielectric function of the composite and showing that carbon nanotubes have an intrinsic and anisotropic metallic behavior. Accurate calculations of the effective dielectric function and the optical response of densely packed carbon nanotubes, as obtained by solving Maxwell's equations with the use of tensorlike dielectric functions, have been carried out only very recently. ${ }^{11}$

In this paper, we extend the calculations reported in Ref. 11 to the case of hollow-core nanotubes, and emphasize the effects of the inner radius on the effective dielectric function of the nanotube array. Calculations of the so-called energyloss function, i.e., the imaginary part of the effective inverse dielectric function, are also presented, for various values of the filling fraction and the ratio of the internal and external radii of the tubules.

We take a periodic array of infinitely long multishell nanotubes of inner and outer radii $r$ and $R$, respectively, arranged in a square array with lattice constant $a=2 x R$, as shown in Fig. 1. These tubules are assumed to be embedded in vacuum. In the energy range 0 to $10 \mathrm{eV}$ of interest in the interpretation of absorption cross sections and energy-loss spectra, the diameter of typical multishell carbon nanotubes $(2 R \sim 10 \mathrm{~nm})$ is small in comparison to the wavelength of light. Hence, our results will be independent of the actual value of the outer radius of the cylinders. We also assume that this radius is large enough that a macroscopic dielectric function is ascribable to the coaxial cylinders. These cylinders occupy a fraction $f=\pi\left(1-\rho^{2}\right) /\left(4 x^{2}\right)$ of space, with $\rho$ $=r / R$ being the ratio between the inner and outer radii of the tubes. For simplicity, the magnetic permeabilities will be assumed to be equal to unity in all media.

Planar graphite is a highly anisotropic material, and the dielectric function is a tensor. This tensor may be diagonalized, by choosing Cartesian coordinates with two of the axis



FIG. 1. Multishell nanotubes of inner and outer radii $r$ and $R$, respectively, arranged in a square array with lattice constant $a$. The cylinders are infinitely long in the $y$ direction. The electromagnetic interaction of this structure with a normally incident plane wave of momentum $\mathbf{k}\left(k_{y}=k_{z}=0\right)$ and energy $\omega$ is investigated. 
lying in the basal plane and the third axis being the so-called $c$ axis. One defines the dielectric function $\varepsilon_{\perp}(\omega)$ perpendicular to the $c$ axis and the dielectric function $\varepsilon_{\|}(\omega)$ for the electric field parallel to the $c$-axis. For carbon nanotubes, we assume full transferability of the dielectric tensor of planar graphite to the curved geometry of carbon tubules, as suggested by Lucas et al. ${ }^{12}$ for the case of multishell fullerenes. Hence, we simply assign a local graphitelike dielectric tensor to every point inside the nanotube and outside the inner core,

$$
\hat{\varepsilon}(\omega)=\varepsilon_{\perp}(\omega)(\theta \theta+\mathbf{z z})+\varepsilon_{\|}(\omega) \mathbf{r r},
$$

$\theta \theta, \mathbf{z z}$, and $\mathbf{r r}$ being the unitary basis vectors of cylindrical coordinates, and take the principal dielectric functions $\varepsilon_{\perp}(\omega)$ and $\varepsilon_{\|}(\omega)$ of graphite from Ref. 13 .

In the long-wavelength limit, a composite material may be treated as if it were homogeneous, with the use of an effective dielectric function $\varepsilon_{\text {eff }}$. The optical absorption cross section of the composite is then directly given by $\operatorname{Im} \varepsilon_{e f f}(\omega)$. Also, for small values of the adimensional parameter $q R(q R<1), \hbar q$ being the momentum transfer, the energy-loss spectra of a broad beam of swift electrons penetrating the composite is found ${ }^{14}$ to be well described by the $q \rightarrow 0$ limit of the imaginary part of the effective longitudinal dielectric function, ${ }^{15}$ i.e., the so-called energy-loss function, $\operatorname{Im}\left[-\varepsilon_{\text {eff }}^{-1}(\omega)\right]$.

In order to compute, with full inclusion of the electromagnetic interaction between the tubules, the effective dielectric function of our periodic system, we first solve Maxwell's equations. The local dielectric function of our composite material depends on frequency. Thus, on-shell methods for solving Maxwell's equations, ${ }^{16}$ which proceed at constant frequency, are particularly well suited for our calculations. Though the methodology of Ref. 16 was originally reported to solve Maxwell's equations with scalar dielectric functions, an extension of this formalism was developed in Ref. 17, which enables us to consider tensorlike dielectric functions like the one of Eq. (1).

We first fix the frequency, thereby the local tensor-like dielectric function at any point of the composite material being specified, and approximate the continuous electromagnetic field by its values at a series of mesh points located on a simple square lattice with unit cell of side $a$, as shown in Fig. 1. The corresponding discretized Maxwell's equations provide a relationship between the electromagnetic fields on either side of the unit cell, i.e., $x$ and $x+a$ (see Fig. 1). We exploit this to calculate, on applying Bloch's theorem, the eigenvalues of the so-called transfer matrix, which will give the band structure of the system, i.e., the dispersion relations $k(\omega)$ of the Bloch-state wave vector of propagation versus the frequency. The effective transverse dielectric function is then obtained as follows,

$$
\varepsilon_{e f f}(\omega)=\frac{k^{2}(\omega) c^{2}}{\omega^{2}},
$$

where $c$ represents the speed of light.

For an electromagnetic wave normally incident on the structure $\left(k_{y}=k_{z}=0\right)$ there are two different values of $\varepsilon_{\text {eff }}(\omega)$ corresponding to $s$ and $p$ polarizations. For electro-

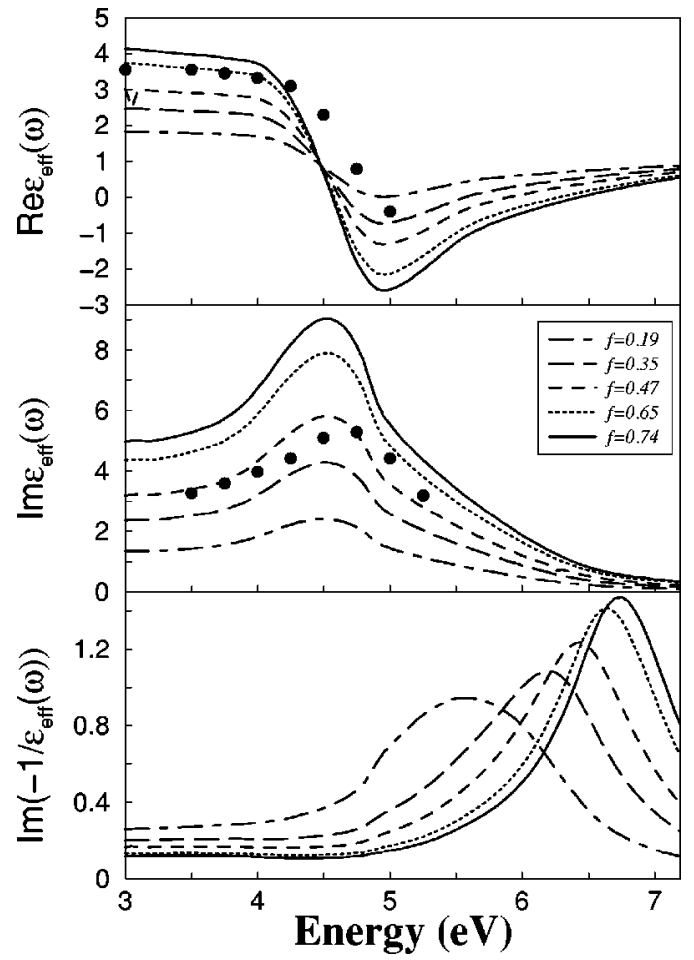

FIG. 2. The real and imaginary parts of the long-wavelength effective dielectric function, $\operatorname{Re} \varepsilon_{e f f}(\omega)$ and $\operatorname{Im} \varepsilon_{e f f}(\omega)$, and the energy-loss function, $\operatorname{Im}\left[-\varepsilon_{e f f}^{-1}(\omega)\right]$, of the periodic system described in Fig. 1, for $s$ polarized electromagnetic excitations. Dashed-dotted, long-dashed, short-dashed, dotted, and solid lines: calculated results, from either Eq. (2) or Eq. (3), for volume filling fractions of $19 \%, 35 \%, 47 \%, 65 \%$, and $74 \%$, respectively. The solid circles represent the experimental results reported in Ref. 9.

magnetic waves polarized along the tubes ( $s$ polarization), the electric field is parallel to the cylinders at every point, and is not modified by the presence of the interfaces. Hence, the effective dielectric function of the composite is simply the weighted average of the dielectric functions of the constituents, ${ }^{18}$

$$
\varepsilon_{e f f}(\omega)=f \varepsilon_{\perp}(\omega)+(1-f) .
$$

Our numerical results for the effective dielectric function, as obtained from Eq. (2) for this polarization, accurately reproduce the exact results predicted by Eq. (3), which represents a good check for our scheme. According to Eq. (3), $\operatorname{Im} \varepsilon_{e f f}(\omega)=f \operatorname{Im} \varepsilon_{\perp}(\omega)$; therefore, the shape of the experimentally determined $\operatorname{Im} \varepsilon_{e f f}(\omega)$ must coincide for this polarization with $\operatorname{Im} \varepsilon_{\perp}(\omega)$. The measurements of $\varepsilon_{\text {eff }}(\omega)$ reported in Ref. 9 for light polarized along the tubes are plotted in Fig. 2 by solid circles, together with the effective dielectric function predicted by Eq. (3), which roughly reproduces with $f \sim 0.5$ the experimentally determined $\operatorname{Im} \varepsilon_{\text {eff }}(\omega)$. We note, however, that neither the exact position of the maximum nor the details of the real part of the measured effective dielectric function can be reproduced with the use of Eq. (3). This may reflect existing differences between carbon struc- 


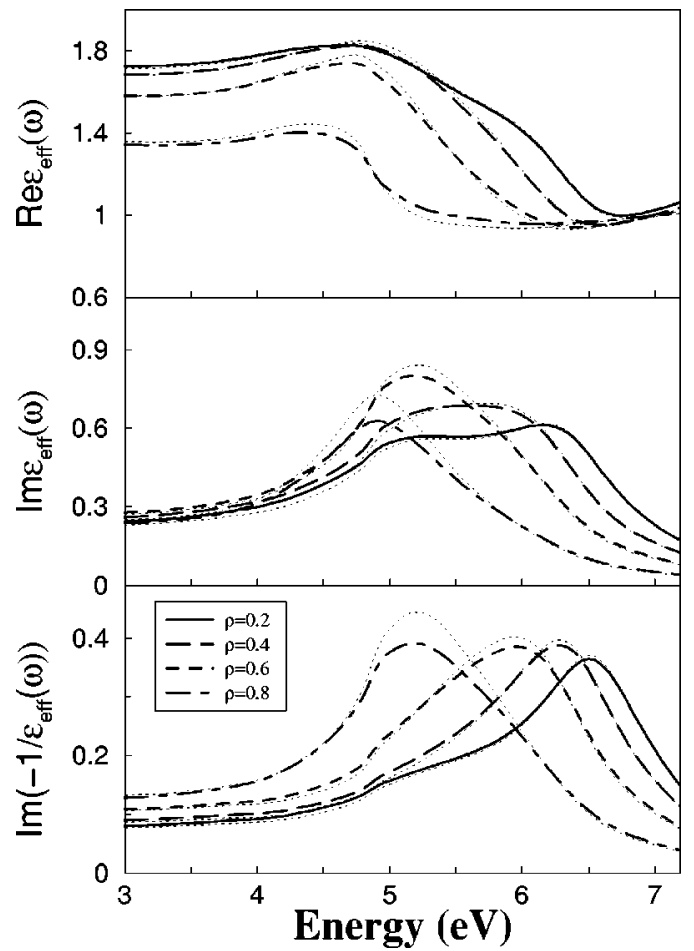

FIG. 3. The real and imaginary parts of the long-wavelength effective dielectric function, $\operatorname{Re} \varepsilon_{e f f}(\omega)$ and $\operatorname{Im} \varepsilon_{e f f}(\omega)$, and the energy-loss function, $\operatorname{Im}\left[-\varepsilon_{\text {eff }}^{-1}(\omega)\right]$, of a periodic array of hollow carbon nanotubes, for $p$ polarized electromagnetic excitations and for the ratio between the lattice constant and the outer diameter of the cylinders $x=2.0$. Solid, long-dashed, short-dashed, and dasheddotted lines represent our full calculations, as obtained from Eq. (2), for ratios between the inner and outer radii of the tubes $\rho$ $=0.2,0.4,0.6$, and 0.8, respectively. The dotted lines represent a generalized MG effective dielectric function appropriate for anisotropic hollow cylinders.

tures in planar graphite and in nanotubes. Also, optical measurements may be affected by the presence of tubes with different orientations.

The effective energy-loss function, $\operatorname{Im}\left[-\varepsilon_{e f f}^{-1}(\omega)\right]$, corresponding to $s$ polarization is also plotted in Fig. 2. For this polarization, the effective inverse dielectric function has a single pole, which occurs at a reduced plasma frequency where $\operatorname{Re} \varepsilon_{\perp} \sim-(1-f) / f$ and $\operatorname{Im} \varepsilon_{\perp} \sim 0$. For graphite, at low concentrations of tubules $(f<0.2)$ the resonance condition is never satisfied, and in the limit as $f \rightarrow 0$ the effective energy-loss function simply coincides with $\operatorname{Im} \varepsilon_{\perp}(\omega)$, thereby showing the characteristic peak at $\sim 4.6 \mathrm{eV}$. This peak is associated with the maximum in the joint density of states of the $\pi$ valence and conduction bands in graphite.

Calculations of the $p$ component of the effective dielectric function of an array of plain $(\rho=0)$ carbon nanotubes were reported in Ref. 11 for various values of the ratio $x$ between the lattice constant and the outer diameter of the cylinders, showing that the trend with increasing the concentration of tubules is for the actual dipolar peak in $\operatorname{Im} \varepsilon_{e f f}(\omega)$ to be shifted from the isolated-cylinder dipole mode at $\sim 6.5 \mathrm{eV}$ to lower energies. When the nanoparticles are brought into close contact, electromagnetic coupling between them con-

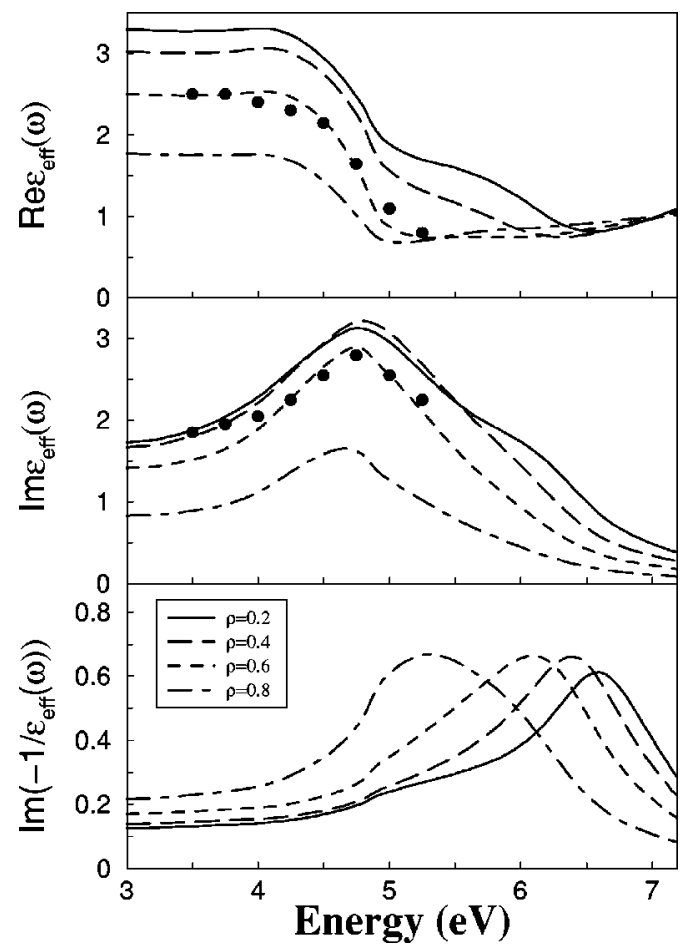

FIG. 4. Same as Fig. 3, for the ratio between the lattice constant and the outer diameter of the cylinders $x=1.03$. The solid circles represent the experimental results reported in Ref. 9.

verts the dipolar surface mode into a very localized one, trapped in the region between the nanostructures. At higher concentrations of tubules, when graphite forms a connected medium $(x \leqslant 1)$, dipolar modes cannot be excited, and the optical absorption exhibits a single peak originated in the maximum of $\operatorname{Im} \varepsilon(\omega)$ at $\sim 4.6 \mathrm{eV}$. We have also calculated the energy-loss function of an array of plain carbon nanotubes, ${ }^{19}$ and have found that for $x \leqslant 1$ it shows a single peak at the bulk plasmon resonance at $\sim 7 \mathrm{eV}$, where $\operatorname{Re} \varepsilon_{\perp}(\omega)$ and $\operatorname{Im} \varepsilon_{\perp}(\omega)$ are both small.

For hollow tubes $(\rho \neq 0)$, there are two distinct dipolar modes with either tangential or radial symmetry, similar to those present in the case of a thin planar film ${ }^{20}$ and a spherical shell. ${ }^{21}$ With the presence of anisotropy this two-mode structure is replaced by a more complicated spectral representation. ${ }^{19}$ Our full calculations of the $p$ effective dielectric function of a periodic array of hollow carbon nanotubes, as obtained for various ratios between inner and outer radii, are shown in Figs. 3 and 4 with $x=2.0$ and $x=1.03$, respectively. $^{22}$ As in the case of plain cylinders $(\rho=0)$, our full calculations nearly coincide for $x=2.0$ with a generalized Maxwell-Garnett (MG) effective dielectric function appropriate for anisotropic hollow tubes. ${ }^{23}$ However, in the close-packed regime $(x=1.03)$ the strong electromagnetic coupling between the tubes yields non-negligible contributions from multipolar resonances. This multipolar coupling provokes a redshift and a blueshift of the MG dipolar resonances that are visible in the optical spectra and the energy loss, respectively. These are the low-energy dipolar mode with tangential symmetry, which absorbs light $\left[\operatorname{Im} \varepsilon_{e f f}(\omega)\right.$ is maximum at the corresponding energy], and the high- 
energy dipolar mode with radial symmetry, which can be excited by moving charged particles $\left(\operatorname{Im}\left[-\varepsilon_{\text {eff }}^{-1}(\omega)\right]\right.$ is maximum at the corresponding energy).

The experimentally determined macroscopic dielectric function of close-packed carbon nanotubes, as reported in Ref. 9 for $p$-polarized light, is exhibited in Fig. 4 by solid circles. A comparison between these measurements and the $p$ effective dielectric function of close-packed $(x=1.03)$ plain $(\rho=0)$ carbon nanotubes was presented in Ref. 11, showing a nice agreement between theory and experiment, though a shift of approximately 1 was needed to obtain agreement with the real part of $\varepsilon_{e f f}(\omega) .{ }^{24}$ If one chooses the ratio $\rho$ between the inner and outer radii of the tubes to be 0.6, which yields in the close-packed regime $(x=1.03)$ a filling fraction $f \sim 0.5$, both real and imaginary parts of our calculated effective dielectric function show excellent agreement with the experiment. This filling fraction $(f \sim 0.5)$ is precisely what is required to also reproduce the dielectric func- tion experimentally determined with the use of light polarized along the cylinders, as discussed above (see Fig. 2).

In conclusion, we have shown that the effective dielectric function of densely packed carbon nanotubes is the result of a strong electromagnetic coupling between the tubes. We have chosen the inner and outer diameters of hollow tubules to be 6 and $10 \mathrm{~nm}$, respectively $(\rho=0.6)$, and have obtained both $s$ and $p$ effective dielectric functions to be, in the closepacked regime $(a=10.3 \mathrm{~nm}, x=1.03)$, in excellent agreement with the experimental measurements. Small discrepancies have been observed in the case of light polarized along the tubes ( $s$ polarization), which may reflect existing differences between the dielectric function of carbon structures in planar and curved graphite.

J.M.P. gratefully acknowledges partial support by the University of the Basque Country, the Basque Hezkuntza, Unibertsitate eta Ikerketa Saila, and the Spanish Ministerio de Educación y Cultura.
${ }^{1}$ S. Ijima, Nature (London) 354, 56 (1991).

${ }^{2}$ T. W. Ebbesen and P. M. Ajayan, Nature (London) 358, 220 (1992).

${ }^{3}$ S. Ijima et al., Nature (London) 356, 776 (1992).

${ }^{4}$ M. S. Dresselhaus, Nature (London) 358, 195 (1992).

${ }^{5}$ J. W. Mintmire et al., Phys. Rev. Lett. 68, 631 (1992).

${ }^{6}$ N. Hamada et al., Phys. Rev. Lett. 68, 1579 (1992).

${ }^{7}$ M. Ge and K. Sattler, Science 260, 515 (1993).

${ }^{8}$ P. M. Ajayan et al., Science 265, 1212 (1994).

${ }^{9}$ W. A. de Heer et al., Science 268, 845 (1995).

${ }^{10}$ F. Bommeli et al., Solid State Commun. 99, 513 (1996).

${ }^{11}$ F. J. García-Vidal et al., Phys. Rev. Lett. 78, 4289 (1997).

${ }^{12}$ A. A. Lucas et al., Phys. Rev. B 49, 2888 (1994).

${ }^{13}$ Handbook of Optical Constants of Solids, edited by E. D. Palik (Academic, New York, 1985).

${ }^{14}$ J. M. Pitarke et al., Phys. Rev. B 55, 9550 (1997); J. M. Pitarke and A. Rivacoba, Surf. Sci. 377, 294 (1997).

${ }^{15}$ In the long-wavelength limit $(q \rightarrow 0)$, longitudinal and transverse dielectric functions with the same polarization coincide.

${ }^{16}$ J. B. Pendry and A. MacKinnon, Phys. Rev. Lett. 69, 2772 (1992); J. B. Pendry, J. Mod. Opt. 41, 2417 (1994).

${ }^{17}$ A. J. Ward and J. B. Pendry, J. Mod. Opt. 43, 773 (1996).
${ }^{18}$ D. J. Bergman and D. Stroud, Solid State Phys. 46, 147 (1992).

${ }^{19}$ F. García-Vidal and J. M. Pitarke (unpublished).

${ }^{20}$ R. H. Ritchie, Phys. Rev. 106, 874 (1957).

${ }^{21}$ A. A. Lucas et al., Nucl. Instrum. Methods Phys. Res. B 96, 470 (1995).

${ }^{22}$ For metallic structures, sampling meshes as large as $180 \times 180$ have been found to be required to provide well-converged results. [J. M. Pitarke et al., Phys. Rev. B 57, 15261 (1998); J. M. Pitarke et al., Surf. Sci. 433, 605 (1999)]. However, for carbon nanotubes sampling meshes of $60 \times 60$ have been found to provide well-converged results, which is due to the smoothing effect of the large damping originated with the presence of interband transitions in graphite.

${ }^{23}$ J. C. Maxwell-Garnett, Philos. Trans. R. Soc. London, Ser. A 203, 385 (1904); 205, 237 (1906); see also C. F. Bohren and D. R. Huffman, Absorption and Scattering of Light by Small Particles (Wiley, New York, 1983).

${ }^{24}$ In Fig. 3 of Ref. 11 the curve labeled (a) for the real part of the effective dielectric function was shifted by a constant value of +4 , while the experimental data for this quantity were shifted by a constant value of +5 . 